(C)2007 IEEE. Personal use of this material is permitted. However, permission to reprint/republish this material for advertising or promotional purposes or for creating new collective works for resale or redistribution to servers or lists, or to reuse any copyrighted component of this work in other works must be obtained from the IEEE 


\title{
Determining the Loss of Investment Probability for Risk based Decision Support System
}

\author{
Omar Khadeer Hussain, Elizabeth Chang, Farookh Khadeer Hussain and Tharam S. Dillon \\ Digital Ecosystem and Business Intelligence Institute, Curtin University of Technology, Perth, Australia \\ Omar.Hussain@postgrad.curtin.edu.au, \{Elizabeth.Chang,Farookh.Hussain,Tharam.Dillon\}@cbs.curtin.edu.au
}

\begin{abstract}
In an e-commerce interaction it is rational for the initiating agent to be apprehensive about the outcome of the interaction and fear for the safety of its resources involved in it. The initiating agent can alleviate this to a certain extent by determining the degree of loss in its resources involved in the interaction. In this paper we propose a methodology by which the initiating agent can ascertain that, and which would further assist it to make an informed decision of its future course of action with an agent.
\end{abstract}

\section{Introduction}

Risk is an omnipresent factor in the modern world e-commerce interactions. It can easily be distinguished from other events due to the unwanted effects associated with it, and its ability to change the outcome of the interaction in a negative way. By specifying 'negative way' we mean the loss, or the occurrence of an undesired outcome in the interaction. In the literature researchers have defined risk by associating it with an unbiased outcome [1]. But the reality is that a positive event might not change the outcome of the interaction that is 'unwanted' in the interacting agent view as much as the negative outcome does, and hence we think that risk associated with the occurrence of a negative outcome. Risk analysis is important in the study of behavior in e-commerce interaction as there is a whole body of literature based in rational economics that argues that the decision to buy is based on the riskadjusted cost-benefit analysis [2]. Risk plays a central role in deciding whether to proceed with a transaction or not. It can broadly be defined as an attribute of decision making that reflects the variance of its possible outcomes. Thus, it commands a central role in any discussion that is related to a transaction.

Trust and Risk are the two concepts associated with an interaction, which complement each other for the interacting agent to make an informed decision of its future course of action with any other agent. Both of these concepts although complement each other, express different meanings, which in turn cannot be reciprocated. Hence it is incorrect to compare and decide as to which one of them is more important for better decision making in an interaction. Gefen et al. reviews and points out the differences between the conceptualization and the relationship between trust, risk and behaviour in the literature [3].

To assess the possible Risk in a context, it is first important to comprehend how risk is interpreted in that particular context. In the literature different definitions has been proposed for risk according to the context in which they are being discussed in [4-7]. Those definitions are defined according to how it fits and best expresses its object of analysis in that context. Similarly risk is assessed according to how it is defined in that discipline. The definition of risk and its assessment method in a context cannot be used to define and assess risk in other disciplines, as the way risk is interpreted and assessed in those disciplines varies and hence would give in-correct conclusions if applied. Therefore in the context of e-commerce interactions or in digital business ecosystem interactions we interpret risk as a multidimensional construct which is a combination of the probability and level of failure of the interaction and the possible consequences of failure [8]. We term the initiating agent and the agent with whom it plans to interact with, as the risk assessing agent and the risk assessed agent respectively. The different dimensions and the levels of risk need to be defined to assess it properly in an interaction. The risk assessing agent, by analyzing the possible risk beforehand in interacting with a risk assessed agent, could determine up to what level it will achieve its desired outcomes in the interaction, and it can also gain an idea or direction in which its interaction might head. Risk management is concerned with making decisions after analyzing the possible risk in an interaction.

Another factor that would assist the risk assessing agent to utilize while determining the possible risk in interacting with a risk assessed agent, is to determine the probability of loss in its investment while 
interacting with that agent. This can be achieved by first ascertaining the level of failure of the risk assessed agent it is going to interact with, and the resources that it is going to invest while interacting with it. In this paper we propose a methodology by which the initiating agent can determine the degree of loss in its investment in an interaction.

\section{Determining the Probability of Failure of the Interaction}

As mentioned earlier, the risk assessing agent has to determine the probability of failure and the possible consequences of failure to its resources in order to analyze the possible level of risk before initiating its interaction with a risk assessed agent. Hence to quantify and represent semantically the probability of failure of a risk assessed agent, we defined the term 'FailureLevel' and the Failure scale in Hussain et al. [8]. FailureLevel quantifies and semantically expresses the possible level of failure on the failure scale. The Failure scale as shown in Figure 1 represents the different levels of failure, possible in a given period of time. The risk assessing agent determines the FailureLevel in interacting with the probable risk assessed agent beforehand by ascertaining its incapability to complete the interaction, according to the expectations of its future interaction with it. In other words, the FailureLevel of an interaction is the extent to which the risk assessing agent determines that it might not achieve its desired outcomes in interacting with a probable risk assessed agent.

The risk assessing agent communicates its desired outcomes and the resources it will invests to achieve them, to the risk assessed agent before interacting with it, in the expected behavior or the mutually agreed behavior. The expectations or the desired outcomes that the risk assessing agent wants in its interaction with a probable risk assessed agent can be classified at a higher level as the 'Context' of the interaction. It can be decomposed into several detailed aspects known as the 'Criteria', which defines the demand or the set of factors which show specifically what the risk assessing agent wants in its interaction with the risk assessed agent in the particular context. Criteria form the expectations or the desired outcomes of the risk assessing agent. By considering its expectations, the risk assessing agent will accurately determine the probability of failure according to its criteria or desired outcomes.

It may be the case that the possible interaction of the risk assessing agent with the probable risk assessed

\begin{tabular}{|c|c|c|c|}
\hline $\begin{array}{l}\text { Semantics of } \\
\text { Failure Level }\end{array}$ & $\begin{array}{c}\text { Probability of } \\
\text { Failure }\end{array}$ & FailureLevels & Star Rating \\
\hline Unknown & . & -1 & Not Displayed \\
\hline Total Failure & $\begin{array}{l}91-100 \% \text { Probability of } \\
\text { Failure }\end{array}$ & 0 & Not Displayed \\
\hline Extremely High & $\begin{array}{l}71-90 \% \text { Probability of } \\
\text { Failure }\end{array}$ & 1 & From 20 to \\
\hline Largely High & $\begin{array}{c}51-70 \% \text { Probability of } \\
\text { Failure }\end{array}$ & 2 & From \\
\hline High & $\begin{array}{c}26-50 \% \text { Probability of } \\
\text { Failure }\end{array}$ & 3 & 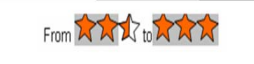 \\
\hline Significantly Low & $\begin{array}{l}\text { 11- } 25 \% \text { Probability of } \\
\text { Failure }\end{array}$ & 4 & From WWW th thW \\
\hline Extremely Low & $\begin{array}{l}0-10 \% \text { Probability of } \\
\text { Failure }\end{array}$ & 5 & 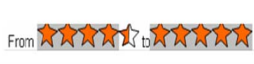 \\
\hline
\end{tabular}

Figure 1: The Failure scale

agent is in the future state of time. Hence, for risk analysis, the risk assessing agent has to determine the FailureLevel in interacting with the probable risk assessed agent in that future state of time. In order to achieve that, we propose that the risk assessing agent analyze the FailureLevel in interacting with a probable risk assessed agent in two stages. They are:

1. Pre-interaction start time phase

2. Post-interaction start time phase

'Pre-Interaction start time phase' refers to the period of time before the risk assessing agent starts its interaction with the probable risk assessed agent, whereas 'Post-Interaction start time phase' is that period of time after the risk assessing agent commences and interacts with the probable risk assessed agent. For risk analysis, the risk assessing agent has to determine the FailureLevel in interacting with a probable risk assessed agent in this period of time, that is in the post-interaction start time phase. However, if this phase is in the future state of time, the risk assessing agent can only determine the FailureLevel by using some prediction methods. So to achieve this, we propose that the risk assessing agent should first ascertain the FailureLevel of the probable risk assessed agent according to the specific context and criteria as that of its future interaction in the preinteraction start time phase. Based on those levels, the risk assessing agent can determine its FailureLevel in the post-interaction start time phase. The determined FailureLevel of the probable risk assessed agent in that time phase depicts the probability and level of failure in interacting with it, during the time of the risk assessing agent's possible interaction with it.

It is possible that the risk assessed agent may have varying levels or degree of failure rather than having a concrete level of failure in a given period of time. Also it is mentioned in the literature that, risk is dynamic varying from time to time. As such, the risk assessing agent should take this dynamic nature of risk into 
consideration while undertaking risk analysis in interacting with a probable risk assessed agent. To incorporate that, we propose the risk assessing agent should divide the total time that it considers to determine the FailureLevel of the probable risk assessed agent, termed as the 'time space', into different non-overlapping parts, termed as 'time slots', and determine the FailureLevel of the risk assessed agent in each of those time slots. By doing so, the risk assessing agent ascertains the correct FailureLevel of the probable risk assessed agent in a time slot, according to its incapability to complete the criterions of its future interaction in that particular time slot, thus considering its dynamic nature while doing risk analysis. The time slots will be spread out either in the pre-interaction or in the post-interaction start time phase. The risk assessing agent has to determine the FailureLevel of the probable risk assessed agent in each time slot according to the time phase in which they fall. The methodology for determining the FailureLevel of the probable risk assessed agent in both the pre- and post-interaction start time phase is defined in Hussain et al [8]. In this paper, we will not discuss the methodology due to space limitation.

The number of time slots in the time space of the interaction depends on the risk assessing agent's duration of the interaction and the division of the time space. Hence it may be the case that the time space is of a very long duration for example 1 year or of a very short duration for example 1 day. What ever the case may be, for risk analysis it would be easier and beneficial for the risk assessing agent if it can analyze the possible risk in interacting with a risk assessed agent for the whole period of the post-interaction start time phase in one go, rather than having to analyze it according to each time slot in that time phase of the interaction. Hence to achieve that we propose that the risk assessing agent after determining the FailureLevel of the risk assessed agent in each time slot of the postinteraction start time phase, should compute its FailureLevel curve. This curve shows the different levels of failures possible while interacting with the risk assessed agent in the post-interaction time phase of the interaction. The abscissa of the curve gives the level of failure and the corresponding ordinate or impulse gives the probability of occurrence of that level.

For better understanding, let us consider an example of risk assessing agent ' $A$ ' wanting to interact with a risk assessed agent ' $\mathrm{B}$ ' in context ' $\mathrm{C}$ ' for a period of 60 days. The criteria that it wants in the interaction are $\mathrm{C} 1$ and $\mathrm{C} 2$. Let us suppose that the risk assessing agent divides the time space into twenty equal time slots with ten in the pre-interaction time phase (t-10 till t-1) and ten in the post-interaction start time phase ( $\mathrm{t} 1$ and $\mathrm{t} 10)$. From the recommendations achieved, the risk assessing agent classifies them according to time, trustworthiness and assimilates them according to the criteria of its future interaction by using the methodology defined in Hussain et al. [8] to determine the FailureLevel of the risk assessed agent in each of the pre-interaction start time slots. Based on the determined FailureLevel of the risk assessed agent in the pre-interaction time slots, the risk assessing agent ascertains the probability of occurrence of the FailureLevel of the risk assessed agent in the post-interaction time phase. From the FailureLevel obtained in each time slot, let us suppose the FailureLevel curve for the post-interaction period from time slot (t1 and t10) is as shown in figure 2.

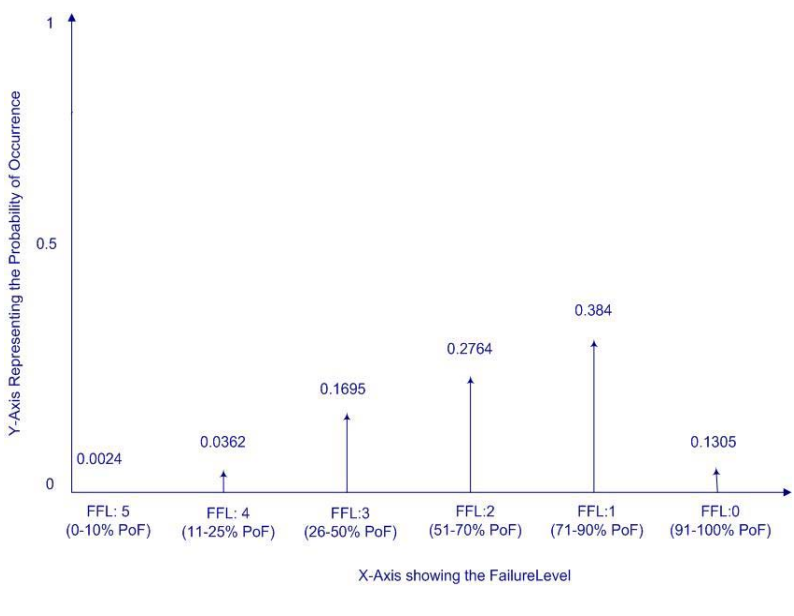

Figure 2: FailureLevel curve of the interaction

The above curve shows the probability of occurrence of each level of failure on the failure scale as determined by the risk assessing agent in interacting with the risk assessed agent in the post-interaction time phase. Once the FailureLevel curve in interacting with a probable risk assessed agent has been determined, the risk assessing agent should then determine the net worth of resources that it going to invest in each of the time slot. In the next sections, we propose a methodology by which the risk assessing agent can ascertain the net worth of resources that it is going to have at stake in the interaction.

\section{Determining the Amount Invested Curve}

The resources invested in an interaction might vary according to the context in which the interaction is being carried out. In this paper we assume that an ecommerce interaction is carried out between the two 
agents and that the risk assessing agent interacts with the probable risk assessed agent to achieve its demand in exchange of the monetary financial value. Subsequently, in our context, the term 'resources' refers to the financial resources invested by the risk assessing agent in its interaction with the risk assessed agent to achieve its demand or desired outcomes. In this paper we are interested in determining the degree of loss in the risk assessing agent's investment. In other terms this is also the degree to which the risk assessing agent will not achieve the full benefit of its resources that it is going to invest in an interaction. To determine that, the risk assessing agent has to first ascertain the probability of the net worth of its resources that it is going to invest throughout the time phase of its interaction.

As mentioned earlier, the number of time slots in the post-interaction phase depends on the risk assessing agent's classification of the time space of the interaction. If there is more than one time slot in the post-interaction phase, then the net worth of the risk assessing agent's resources at stake in the interaction increases progressively as the time slots increase according to the total worth invested in each of them. We are interested in determining the resources invested by the risk assessing agent only in the post-interaction phase of the interaction, as this is the phase in which the risk assessing agent will interact with the risk assessed agent and hence will put its resources at stake. To determine the net worth of its resources invested in the interaction let us consider our previous discussion of the risk assessing agent ' $A$ ' wanting to interact with a risk assessed agent ' $B$ '. In its interaction there are ten time slots $(\mathrm{t} 1-\mathrm{t} 10)$ in the post-interaction time phase and let us suppose that the risk assessing agent ' $\mathrm{A}$ ' invests $\$ 20,000$ in the interaction over those time slots. Two possibilities arise in the nature of the risk assessing agent investing its resources in the interaction. Either it is possible that the risk assessing agent invests the maximum threshold of its resources at once in the beginning of a time slot, or may invest it progressively, in stepwise way in each time slot. For example, it is possible that the risk assessing agent invests $\$ 20,000$; the total net worth of its resources in the first time slot, or it may invest the total worth of its resources progressively, that is say in the order of $\$ 2,000 ; \$ 3,000 ; \$ 6,000 ; \$ 5,000$ and $\$ 4,000$ in time slots $1,3,4,6$ and 9 of the post-interaction time phase respectively to gradually make the total worth of the interaction $\$ 20,000$. In both cases, the probability of an amount invested from its resources throughout the interaction is different. In the first case, when the risk assessing agent invests $\$ 20,000$ at the beginning of the first time slot, the net amount of its resources that it has at stake, throughout the time phase is $\$ 20,000$. On the other hand, if the resources were invested in a stepwise way in each time slot as explained earlier, then the total worth of its resources at stake reaches $\$ 20,000$ on time slot 19 of the time phase. This means that the risk assessing agent has $\$ 20,000$ worth of resources at stake for only 2 time slots of the time phase as compared to having $\$ 20,000$ at stake in all the time slots in the first case. Hence, the risk assessing agent, according to the investment nature of its resources should first ascertain the probability of the net worth of its resources at stake throughout the time space.

To achieve that, we propose the calculation of an Amount Invested Curve (AIC). This curve gives the probability of an amount invested and at stake throughout the duration of the interaction from the invested resources of the risk assessing agent, to achieve its desired outcomes in interacting with a probable risk assessed agent, as initially decided in the expected or mutually agreed behavior. Another important property of this probabilistic model of the amount invested curve is that it describes the probability of the worth of the interaction to be at least a certain amount throughout the duration of the time phase. To calculate the amount invested curve (AIC) we utilize the Fundamental Probability Formulae to determine the probability of an amount being invested throughout the time phase.

To obtain the amount invested curve for our example, let us consider that the risk assessing agent invests $\$ 20,000$ in the interaction in a stepwise way as explained earlier. Determining and representing in figure 3 the amount invested curve for the interaction by using the probability function formulae.

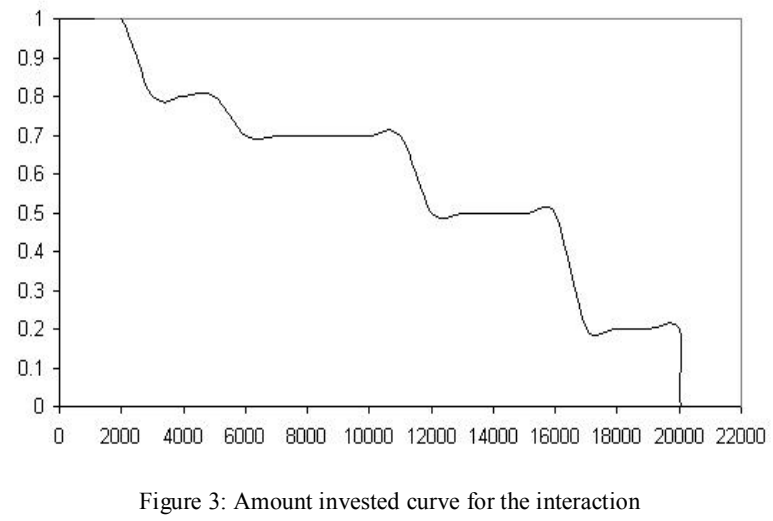

However, it is possible that a risk assessing agent might not achieve its full desired outcomes according to its expectations, in interacting with a probable risk 
assessed agent due to its FailureLevel. Alternately, it can be stated that the risk assessing agent might not get the full benefit of its resources that it invests in an interaction while interacting with a probable risk assessed agent to achieve its desired outcomes, due to its FailureLevel. The degree to which the risk assessing agent might not get the full benefit of its resources invested in an interaction depends on the magnitude of the FailureLevel of the risk assessed agent. The higher the magnitude of failure of the probable risk assessed agent, the higher the probability of loss in the risk assessing agent's resources and vice versa. The risk assessing agent, by determining the degree of loss in its investment in interacting with a probable risk assessed agent, can utilize it to determine the possible risk in interacting with it, and also can make a better informed decision of choosing which agent to interact with among a set of possible risk assessed agents. In the next sections, we will propose a methodology by which the risk assessing agent can determine the degree of loss in its resources in interacting with a probable risk assessed agent.

\section{Determining the Factual Amount Invested Curve}

To ascertain and quantify the degree of loss in the investment of the risk assessing agent, we propose the calculation of the Factual Amount Invested Curve (FAIC). The factual amount invested curve shows the required probability of an amount to be kept at stake by the risk assessing agent, throughout the duration of the interaction to achieve its desired outcomes, by taking into consideration the FailureLevel of the risk assessed agent and the probability of that amount it was initially investing. Hence, the factual amount invested curve (FAIC), which shows the increased probability of an amount that the risk assessing agent needs to invests in an interaction, is an extension of the amount invested curve (AIC). The AIC shows the actual probability of an amount invested and at stake from the resources of the risk assessing agent throughout the duration of the interaction, according to the expected or mutually agreed behavior, whereas the FAIC shows the required probability of the risk assessing agent to invest that amount throughout the duration of the interaction by considering the FailureLevel of the risk assessed agent.

To obtain the FAIC of an interaction, the AIC should be convolved with the FailureLevel curve of the risk assessed agent. We utilize the cumulants method for the convolution of these two functions. In this method, the convolution of the independent random variables can be expressed as a sum of their individual cumulants, which can then be used to model the output curve, which is the FAIC by using either GramCharlier series expansion or Beta distribution. In our problem, an advantage of using this method over the conventional point by point method for convolution is that, in the cumulants method the convolution of the independent random variables can be determined as a sum of their individual cumulants, whereas in the conventional method it is necessary to divide the AIC into different parts and then convolve it with the density function of the FailureLevel curve of the risk assessed agent. The output of the convolution, which is the FAIC, is an inflated curve as compared to the AIC. This curve is inflated as it shows the increased probability of an amount that the risk assessing agent needs to invest in the interaction.

Considering the previous example, the risk assessing agent ' $A$ ' can determine the FAIC in time slots $\mathrm{t} 1 \mathrm{-} \mathrm{t} 10$, by convolving the FailureLevel curve of the risk assessed agent in those time slots, represented in figure 2 with the AIC of those time slots represented in figure 3. The resultant inflated FAIC of the interaction is given in figure 4 .

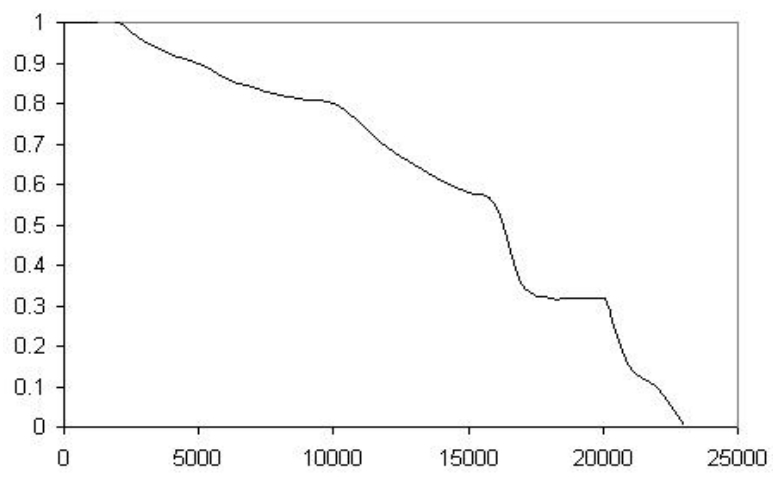

Figure 4: Factual Amount invested curve for the interaction

Once the risk assessing agent determines the FAIC, it can then determine the degree of loss in its investment or resources while interacting with the risk assessed agent.

\section{Determining the Loss of Investment Probability in an Interaction}

The Loss of Investment probability (LOIP) of an interaction can be determined by ascertaining the level to which the risk assessed agent will not complete the desired outcomes of the risk assessing agent in the interaction, in the financial resources it initially promised during the expected or the mutually agreed behavior. Alternately the LOIP index of an interaction 
gives the probability of the risk assessing agent not achieving the full benefit of its resources invested, due to the risk assessed agent not completing its desired outcomes, in the amount initially promised according to the expected behavior or the mutually agreed behavior. Hence, LOIP index of an interaction is simply the ordinate on the FAIC, at the end of net resources invested in the interaction, i.e. at the end of AIC. By definition of FAIC, this ordinate is the probability of that amount needed to be at stake in the interaction, but this amount will not invested by the risk assessed agent as it is more than what was initially agreed upon. Hence

$$
\text { LOIP }=\text { FAIC }(w)
$$

where, $w$ is the abscissa at the end of AIC, and

FAIC $(w)=$ Factual amount invested curve after investing the total resources of the interaction.

To explain better, at the end of the AIC the risk assessing agent expects to achieve its desired outcomes of the interaction according to the expected or mutually agreed behavior, in the resources it invested. FAIC shows the required probability of an amount to be invested by the risk assessing agent to achieve its same desired outcomes, by considering the FailureLevel of the risk assessed agent in the interaction. As opposed to what was promised initially, it is possible that at the end of AIC, the risk assessing agent still has some desired outcomes to achieve, due to the FailureLevel of the risk assessed agent. Subsequently, the ordinate on the FAIC immediately at the end of AIC shows the LOIP index of the interaction. In other terms this shows the risk assessing agent not achieving the full benefit or expected returns of its resources that it has invested, due to its unachieved desired outcomes.

Extending the previous example, the risk assessing agent ' $A$ ' can utilize the methodology to determine the loss of its investment probability (LOIP) in interacting with the risk assessed agent ' $\mathrm{B}$ ' in the time slots of its interaction. In the post-interaction time phase of its interaction the risk assessing agent ' $A$ ' invests its resources in a stepwise way to achieve its desired outcomes. But as shown in figure 5 at the end of the net resources invested, that is, at the end of AIC, the LOIP index of the interaction is 0.32 , which means that there is $32 \%$ probability of loss in the risk assessing agent's resources invested in the interaction due to its FailureLevel and as a result un-fulfillment of its desired outcomes, as opposed to what was promised initially. The risk assessing agent can utilize the LOIP index of the interaction, when it is determining the possible risk in interacting with the risk assessed agent

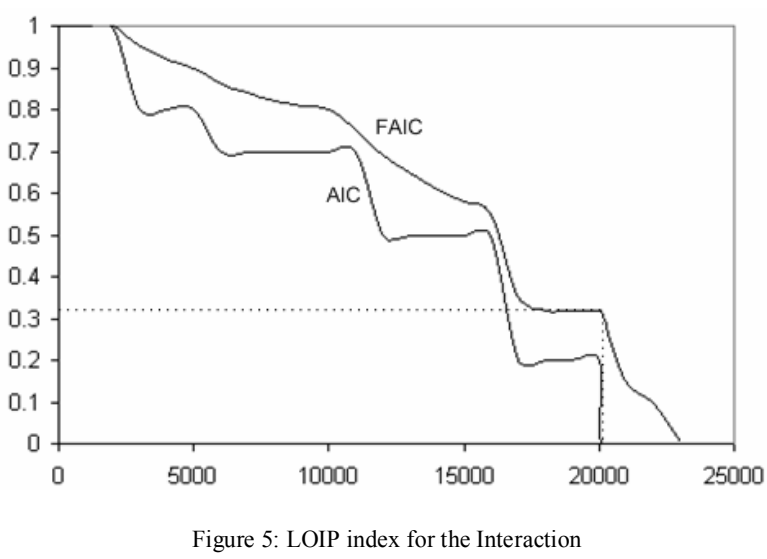

hence enabling it to make a better informed decision while deciding its future course of action with it.

\section{Conclusion}

In this paper we proposed a methodology by which the risk assessing agent can ascertain beforehand the probability of loss in its investment that it is going to put at stake while interacting with a probable risk assessed agent. The risk assessing agent can utilize the LOIP index apart from considering the probability of failure and the possible consequences of failure in interacting with a risk assessed agent, to determine the possible risk in interacting with it. This would help the risk assessing agent to make an informed decision of its future course of action with that agent.

\section{References}

[1] R.C. Mayer, J.H. Davis and F.D. Schoorman, "An interactive model of organizational trust", Academy of Management Review, vol. 20 , no. 3 , 1995, pp.709-734.

[2] S. Greenland, "Bounding analysis as an inadequately specified methodology", Risk Analysis vol. 24, no. 5, 2004, pp. 1085-1092.

[3] D. Gefen, V. S. Rao, N. Tractinsky, "The Conceptualization of Trust, Risk and Their Relationship in Electronic Commerce: The Need for Clarifications", Proceedings of the $36^{\text {th }}$ Hawaii international Conference on System Sciences (HICSS), Hawaii, 2003, pp. $192-201$.

[4] J.G. March and Z. Shapira, "Managerial perspective on risk and risk taking", Management Science, vol. 33, no. 11, 1987, pp. 14041418 .

[5] N. Luhmann, "Familiarity, confidence, trust: Problems and alternatives", Trust: Making and Breaking Cooperative Relations, Basil Blackwell, New York, USA, 1988.

[6] D.M. Rousseau, S.B. Sitkin, R.S. Burt and C. Camerer, "Not so different after all: A cross-discipline view of trust", Academy of Management Review, vol. 23, no. 4, 1998, pp. 393-404.

[7] P. Sztompka, "Trust: A sociological theory", Cambridge University Press, Cambridge, U.K., 1999.

[8] O. K. Hussain, E. Chang, F. K. Hussain and T. S. Dillon, 'A methodology to quantify failure for risk-based decision support system in Digital Business Ecosystems', Accepted for Publication in Data \& Knowledge Engineering, Elsevier Science, To be published in April 2007. 